\title{
Lasting Prolonged-Release Tapentadol for Moderate/ Severe Non-Cancer Musculoskeletal Chronic Pain
}

\author{
Boaz G. Samolsky Dekel • Sivia Ghedini • Alberto Gori • \\ Alessio Vasarri $\cdot$ GianFranco Di Nino $\cdot$ Rita M. Melotti
}

To view enhanced content go to www.paintherapy-open.com

Received: November 26, 2014 / Published online: January 6, 2015

(c) The Author(s) 2014. This article is published with open access at Springerlink.com

\section{ABSTRACT}

Introduction: Despite opioids' recognized role in the treatment of moderate/severe musculoskeletal chronic pain, their long-term benefits need investigation. We explored the lasting analgesic efficacy, tolerability, influence on life quality, and chronicity stage of the novel prolonged release (PR) opioid, tapentadol, in 30 outpatients.

Methods: We evaluated patients' pain intensity and relief (Numerical Rating Scale; NRS), adverse effects, sleep quality, treatment satisfaction, health status (12-questions

B. G. Samolsky Dekel ( $₫)$ · S. Ghedini ·

G. Di Nino - R. M. Melotti

Department of Medicine and Surgery Sciences,

University of Bologna, Via Massarenti n. 9,

40138 Bologna, Italy

e-mail: boaz.samolskydekel@unibo.it

B. G. Samolsky Dekel · S. Ghedini · G. Di Nino ·

R. M. Melotti

Azienda Ospedaliera-Universitaria di Bologna

Policlinico S. Orsola-Malpighi, Via Massarenti n. 9,

40138 Bologna, Italy

B. G. Samolsky Dekel · A. Gori · A. Vasarri ·

G. Di Nino - R. M. Melotti

Post Graduate School of Anaesthesia and Intensive

Care, University of Bologna, Via Massarenti n. 9,

40138 Bologna, Italy
Health-Survey; SF-12), chronicity stage (Italian Mainz Pain-Staging System; I-MPSS) at 10, 30, 60 , and 90 days after tapentadol prescription.

Results: At follow-ups, the investigated outcomes showed an overall statistically significant (Wilcoxon signed-rank test) improvement and remained stable over time, as did the health status and chronicity stage. Adverse effects were limited, transitory, and tolerable.

Conclusions: Twelve weeks of PR tapentadol in outpatients with moderate/severe chronic musculoskeletal pain showed satisfactory analgesic efficacy and tolerability, and had a positive influence on life quality and chronicity stage. The results are robust enough to warrant a subsequent study with a larger sample and a longer observation period.

Keywords: Chronicity; Chronic pain;

Long-term opioids; Musculoskeletal;

Quality of life; Tapentadol

\section{INTRODUCTION}

Moderate/severe non-cancer chronic pain (CP) occurs in $19 \%$ of European adults, affecting 
their daily, social, and working lives; most patients do not receive pain treatment, and 40 $\%$ receive inadequate pain treatment [1].

Chronic pain represents a challenge for orthopedists and pain specialists given its multiple nature which produces both physical and psychological suffering, and because the underlying complex pathophysiological mechanisms require individualized management and a pharmacological approach $[2,3]$. The lack of individually tailored management may lead to inappropriate treatments, useless analgesic dose escalations, and the failure of multiple therapies; these may result in ineffective pain control, harmful adverse effects, low patient compliance, therapy discontinuation, and increased healthcare costs, patient frustration, and suffering [2-4]. The main goals of CP pharmacological management are pain control with a satisfactory quality of life (QoL) and functional and social recovery $[4,5]$ both in the short and long term.

Opioids have a recognized role in the treatment of moderate/severe non-cancer $\mathrm{CP}$; however, their lasting benefits in various $\mathrm{CP}$ conditions and in terms of tolerability and QoL still need further clinical investigation $[6,7]$. Many of these issues directly depend upon the $\mu$-receptor agonist activity of opioids. Indeed, the endogenous opioid system is physiologically implicated in several vital functions and homeostatic systems (e.g., respiratory, gastrointestinal, hormonal, and immune milieus). Thus, along with analgesia, opioids may hamper these systems and thereby induce adverse side effects (e.g., nausea, constipation, immunodepression, and opioid-induced androgen-deficiency) that in the long term may compromise patients' health conditions and QoL.

To address the need for efficacious, safe, and well-tolerated analgesics, a new drug, namely prolonged release (PR) tapentadol, with a dual mode of action ( $\mu$-opioid-receptor agonist and norepinephrine reuptake inhibitor) has been developed; it is reported to produce both satisfactory analgesia and a better tolerability profile due to its limited opioid component [8-12]. Evidence shows that it is efficacious and tolerated in patients with cancer and non-cancer CP [6, 13-22].

We sought to explore, in non-cancer outpatients with musculoskeletal CP and over a period of 12 weeks, the lasting PR tapentadol analgesic efficacy, tolerability profile, and influence on QoL and chronicity stage as a first step towards a future trial in which the long-term efficacy and tolerability profile of PR tapentadol in such CP patients will be evaluated.

\section{METHODS}

\section{Settings and Patients}

This observational study was held at the Acute and CP center of Bologna's Teaching Hospital, Italy. The center is an anesthesiology-based pain program that provides outpatient consultation for primary care physicians and specialty services for inpatients. The sample included 30 consecutive mixed non-cancer CP outpatients who were treated with tapentadol for at least 90 days.

\section{Proceedings, Instruments, and Rationale}

The aims of the study were to explore the lasting analgesic efficacy, tolerability profile, and influence on patients' QoL and chronicity stage of PR tapentadol. Upon recruitment (V0) and after signed informed consent, patients went through a thorough physical examination and history taking for $\mathrm{CP}$ 
diagnosis; information on current pain intensity, medications, and their doses was also obtained. Other investigated variables were: primary pathology, gender (male/ female), and age groups (patients were divided into 20-year interval groups: 30-50, $51-70,>70$ years). At the follow-ups $(10,30$, 60 , and 90 days after V0, respectively) we collected information on the ongoing pain therapy, tapentadol dose variations, analgesic efficacy, tolerability, and patient satisfaction; the General Health Status and chronicity stage, by means of the 12-questions Health-Survey (SF12) and the Italian Mainz Pain-Staging System (I-MPSS) questionnaires, were evaluated at V0 and V90, respectively. The SF-12 provides a multi-dimensional profile of health status and two summary scores assessing physical function and mental well-being; the I-MPSS, recently validated [23], is a measure of pain chronicity and classifies patients at three chronicity stages (I mild; II moderate; and III severe). The rationale for exploring the variables used in this study was that they all have strong relevance to the issue of long-term benefits of opioids in CP conditions both in terms of efficacy, tolerability, health status, and QoL.

If patients were not available for an office visit, follow-ups were made by phone. Table 1 reports the investigated variables and tools used for data collection.

Prolonged release tapentadol was administered and titrated according to the therapeutic indications, the dosages and the instructions given in the summary of the product characteristics and evidence reported in the literature [19, 24-27]. In opioid-naïve patients, PR tapentadol starting dose was $50 \mathrm{mg}$ 12-hourly; doses were then titrated, with increments of $50 \mathrm{mg}$ 12-hourly, each visit as needed (within the therapeutic daily dose range of $100-500 \mathrm{mg}$ ). Titration proceeded until patients achieved at least a 2-point decrease in their Numerical Rating Scale (NRS) pain score from V0; the latter was defined as a clinically relevant improvement in pain relief and was the minimum target of titration. Titration continued until at least the minimum target of titration was reached or the patient was taking PR tapentadol $500 \mathrm{mg}$ daily. For opioid-tolerant patients, previous opioid medication was replaced with equianalgesic doses of $P R$ tapentadol. In these patients, PR tapentadol starting doses were based on morphine equivalent doses (MED): for an average daily MED of $\leq 100, \quad 101-160, \quad$ or $>160 \mathrm{mg}, \quad P R$ tapentadol starting doses were 50, 100, or $150 \mathrm{mg}$ 12-hourly, respectively; dose titration and increments hence followed the scheme illustrated for opioid-naïve patients. During the study, patients were not allowed to use other opioids, pain killers, or adjuvant medications.

We have hypothesized that to have lasting analgesic efficacy, an acceptable tolerability profile, and a positive influence on patients' QoL and chronicity stage, the measured outcomes should show statistically significant stable improvement over the study time frame, limited dropouts $(<10 \%)$, and tolerable adverse effects.

\section{Compliance with Ethics Guidelines}

The study was approved and authorized by the hospital's ethics committee. All procedures followed were in accordance with the ethical standards of the responsible committee on human experimentation (institutional and national) and with the Helsinki Declaration of 1975, as revised in 2000 and 2008, and was conducted according to the International Association for the Study of Pain (IASP)'s guidelines for pain research in animals and 
Table 1 Investigated variables and tools used for data collection

Domain
Primary pathology and concomitant pathologies

Pain therapy upon recruitment

Tapentadol doses at V0 and at follow-ups

Pain characteristics

Pain intensity

Pain relief

Patient satisfaction

Sleep quality

Treatment tolerability

General health status

Chronicity stage

\section{Tools and variable subsets}

Patients' clinical history, physical exam, clinical evidence (imaging, etc.)

Drug classes: none; paracetamol; NSAIDs; mild or strong opioids; adjuvants

Administration schedule: ATC, PRN

Mean daily dose, $\mathrm{mg} / 24-\mathrm{h}$; proportions of patients with 50,100 , and $150 \mathrm{mg} / 12$-hourly

Pain localization: cervical spine, upper limbs, lumbar spine, lower limbs, trunk, articulations, other (specify)

Number of pain sites: One, two, three, diffused pain

Pain duration: $0-3,3-6,6-12,>12$ months

Pain onset cause: Spontaneous at rest; evoked by standing up; evoked by movement, ambulation, coughing, inspiration, sphincteral activity; other (specify)

Pain temporal patterns: Intermittent, continuous, episodic

$0-10$, NRS $(0=$ no pain, $10=$ worse pain I can imagine)

$0-10$, NRS $(0=$ no relief at all, $10=$ complete relief $)$

$1-7$, NRS ( $1=$ extremely improved, $4=$ stationary, $7=$ extremely worsened)

"In the last week your sleep was": $1=$ profound; $2=$ good; $3=$ with frequent wake-ups; $4=$ very disturbed

In the advent of asthenia, vomiting, nausea, itching, dizziness, poor appetite, dysuria, constipation, headache; report for each: date, severity (mild, moderate, severe), and actions taken (none, dose reduction, therapy discontinuation, other)

SF-12 which produces PCS and MCS

I-MPSS which classifies patients at three chronicity stages (I mild; II moderate; and III severe CP)

Adjuvants $=$ anti-convulsants, anti-depressants

$A T C$ around the clock, $C P$ chronic pain, I-MPSS 10-items Italian Mainz Pain-Staging System, MCS mental component summary, NRS Numerical Rating Scale, NSAIDs non-steroidal anti-inflammatory drugs, PSC psychometrically based physical component summary, $P R N$ on demand, $S F-12$ 12-items health survey 
humans. All participants were informed of the study aims and structure and that participation was voluntary, anonymous, and would not affect their care. Informed consent was obtained from all patients for inclusion in the study.

\section{Data Presentation and Statistical Analysis}

Analyses were conducted using StatView for Windows (SAS Institute Inc., Cary, NC, USA) Continuous data were reported as the mean ( \pm standard deviation). The NRS (on a 0-10 scale) and other ordinal data were reported as the median [95\% upper and lower confidence intervals (CI) and interquartile range (IQR)]. Category data and proportions were expressed in percentages. A comparison between different follow up outcomes was made using the nonparametric Wilcoxon signed-rank (WSR) test. Statistical significance was defined as $P<0.05$. When appropriate, $P<0.01$ and $P<0.001$ were reported.

\section{RESULTS}

Of the 30 patients who completed the 90-day follow-up, $66 \%(n=20)$ were women; the mean age of the sample was $72.5( \pm 13.6)$ years (range 33-88). Table 2 summarizes the main clinical features of the sample at VO. In the majority of cases the primary pathology was lumbago with or without sciatica (64\%); pain was localized at one site (lower limbs $53 \%$ ), spontaneous at rest (47\%), and had a duration of $>12$ months (77 $\%)$. Multiple pain medications were reported by $50 \%$ of patients, and adjuvant ones (e.g., pregabalin, gabapentin, and tricyclic antidepressants) by $33 \%$. The majority of patients $(76.6 \%)$ reported further health issues
Table 2 Major characteristics of the sample at V0

\begin{tabular}{|c|c|}
\hline Variable & $n(\%)^{\mathrm{a}}$ \\
\hline \multicolumn{2}{|l|}{ Primary pathology } \\
\hline Lumbago and sciatica & $11(37)$ \\
\hline Lumbar spine & $8(27)$ \\
\hline Cervico-brachialgia & $5(17)$ \\
\hline Knee arthritis & $4(13)$ \\
\hline Vertebral arthritis & $3(7)$ \\
\hline Diabetic neuropathy & $2(7)$ \\
\hline Arthritis and fibromyalgia & $1(2)$ \\
\hline \multicolumn{2}{|l|}{ Pain localization } \\
\hline Lower limbs & $16(53)$ \\
\hline Lumbar spine & $11(37)$ \\
\hline Upper limbs & $5(17)$ \\
\hline \multicolumn{2}{|l|}{ Number of pain sites } \\
\hline One & $16(53)$ \\
\hline Two & $9(30)$ \\
\hline Three & $1(3)$ \\
\hline Diffused pain & $4(13)$ \\
\hline \multicolumn{2}{|l|}{ Pain onset } \\
\hline Spontaneous at rest & $14(47)$ \\
\hline Evoked by standing up & $7(23)$ \\
\hline Evoked by movement & $9(30)$ \\
\hline \multicolumn{2}{|l|}{ Pain duration } \\
\hline$>12$ months & $23(77)$ \\
\hline $3-6$ months & $5(17)$ \\
\hline$<3$ months & $2(7)$ \\
\hline \multicolumn{2}{|l|}{ Temporal patterns } \\
\hline Intermittent & $16(54)$ \\
\hline Continuous & $10(33)$ \\
\hline Episodic & $4(13)$ \\
\hline \multicolumn{2}{|l|}{ Pain therapy } \\
\hline None & $5(17)$ \\
\hline Paracetamol $^{\mathrm{b}}$ & $10(33)$ \\
\hline NSAIDs $^{c}$ & $5(17)$ \\
\hline Opioids ${ }^{\mathrm{d}}$ & $13(43)$ \\
\hline \multicolumn{2}{|c|}{$\begin{array}{l}\text { ATC around the clock, NSAIDs non-steroidal anti-inflammatory } \\
\text { drugs } \\
\text { a The number of cases and proportions may exceed } n=30 \% \text { and } \\
100 \% \text { due to the multiple presence of different variable subsets in the } \\
\text { same patient } \\
\text { b Alone or in association with codeine or tramadol; regularly ATC } \\
\text { in } n=9 \text {, and occasionally in } n=1 \\
\text { c Regularly ATC } \\
\text { d ATC in } n=7 \text {, and occasionally in } n=6\end{array}$} \\
\hline
\end{tabular}


with a range of one to five in the same patient. The most frequent pathology was hypertension (43.3\%) followed by constipation, diverticulosis, and esophagitis.

Table 3 reports the mean of tapentadol daily dose, the number of adverse effects, the median and interquartile range of pain intensity and relief, patient satisfaction, and quality of sleep from V0 to V90. Pain intensity significantly decreased from V0 to V10 and remained stable over time. Indeed, the WSR test showed statistically significant differences between V0V10 $(P=0.0003)$, V0-V30, V0-V60, and V0V90 ( $P=0.0001$, respectively); significant differences were also shown between V10-V30 $(P=0.006)$, but not between V30-V60 and V60-V90 ( $P>0.05$, respectively), indicating stable pain reduction from the thirtieth day onwards.

Pain relief improved from V10 to V30 and remained stable over time. Indeed, the WSR test showed statistically significant differences between V10-V30 $(P=0.007)$, but not between V30-V60 and V60-V90 $(P>0.05$, respectively), indicating stable pain relief from the thirtieth day onwards.

Sleep quality significantly improved from V0 to V10 and remained stable over time. Indeed, the WSR test showed statistically significant differences between V0-V10 $(P=0.036)$, V0V30, V0-V60, and V0-V90 $(P=0.043$, $P=0.011, P=0.018$, respectively); significant differences were not shown between V10-V30, V30-V60, and V60-V90 ( $P>0.05$, respectively), indicating stable good sleep quality from the tenth day onwards.

For the SF-12 evaluations, psychometrically based physical (PCS) and mental (MCS) component summaries showed improvements from V0 to V90 (Table 3). The WSR test showed statistically significant differences for PCS $(P=0.006)$, but not for MCS $(P=0.091)$.
Interestingly, while MCS showed limited improvement, its IQR considerably decreased from V0 to V90. When split by gender PCS and MCS differences between V0 and V90 were statistically significant for men (WSR, $P=0.025$ and $P=0.049$, respectively), but not for women. When split by age groups PCS differences between V0 and V90 were statistically significant among patients of $>70$ years.

Proportions of chronicity stages, evaluated with the I-MPSS questionnaire, showed an improvement trend from V0 to V90. Indeed, as shown in Table 3, proportions of stage I (low chronicity) increased from 13.3 to $40.0 \%$ and those of stage III (high chronicity) decreased from 40.0 to $20.0 \%$.

\section{DISCUSSION}

In this study we explored the lasting PR tapentadol analgesic efficacy, tolerability profile and influence on QoL and chronicity stage in a cohort of non-cancer CP outpatients as a first step towards a future trial in which the long-term efficacy and tolerability profile of PR tapentadol in such CP patients will be evaluated. In agreement with other studies [6, 13-19, 22], our data show overall satisfactory outcomes in CP outpatients treated with PR tapentadol over a period of 90 days. These results offer several clinically useful observations.

Tapentadol was chosen as it was reported to produce both satisfactory analgesia and, vs. other strong opioids, a better tolerability profile due to its limited opioid component [8-12]. Indeed, evidence and its dual mode of action ( $\mu$-opioid-receptor agonist and norepinephrine reuptake inhibitor) offer a solid rational for it to be an efficacious, safe, and well-tolerated analgesic in patients with cancer and non-cancer CP [19-22]. 
Table 3 Outcome evaluations from V0 to V90

\begin{tabular}{|c|c|c|c|c|c|}
\hline Outcome & Vo & V10 & V30 & V60 & V90 \\
\hline \multicolumn{6}{|l|}{ Tapentadol dose } \\
\hline Daily dose $(\mathrm{mg})$, mean $( \pm \mathrm{SD})$ & $126.7( \pm 58.3)$ & $180.0( \pm 71.4)$ & $186.7( \pm 68.1)$ & $193.3( \pm 74.0)$ & $193.3( \pm 74.0)$ \\
\hline 50 mg/12-hourly, $n$ (\%) & $24(80.0)$ & $11(36.7)$ & $9(30.0)$ & $9(30.0)$ & $9(30.0)$ \\
\hline 100 mg/12-hourly, $n$ (\%) & $4(13.3)$ & $14(46.7)$ & $16(53.3)$ & $14(46.7)$ & $14(46.7)$ \\
\hline $150 \mathrm{mg} / 12$-hourly, $n$ (\%) & $2(6.7)$ & $5(16.7)$ & $5(16.7)$ & $7(23.3)$ & $7(23.3)$ \\
\hline Pain intensity $(\mathrm{NRS})^{\mathrm{a}}$ & $6.0(2.0)$ & $4.0(3.0)$ & $3.0(3.0)$ & $3.0(4.0)$ & $2.0(4.5)$ \\
\hline Pain relief $(\mathrm{NRS})^{\mathrm{a}}$ & & $5.0(6.0)$ & $7.0(5.5)$ & $7.0(7.0)$ & $7.5(6.0)$ \\
\hline Patient satisfaction $(7 \text { items })^{\mathrm{a}}$ & & $5.0(2.0)$ & $6.0(1.5)$ & $6.0(2.0)$ & $6.0(1.5)$ \\
\hline Sleep quality $(4 \text { items })^{\mathrm{a}}$ & $3.0(2.0)$ & $4.0(1.5)$ & $4.0(2.0)$ & $4.0(1.0)$ & $4.0(1.0)$ \\
\hline \multicolumn{6}{|l|}{ SF-12 } \\
\hline $\mathrm{PCS}^{\mathrm{a}}$ & $29.7(11.4)$ & & & & $35.8(18.1)$ \\
\hline $\mathrm{MCS}^{\mathrm{a}}$ & $52.4(19.1)$ & & & & $53.5(3.2)$ \\
\hline \multicolumn{6}{|l|}{ Chronicity stage [I-MPSS, $n(\%)]$} \\
\hline $\mathrm{I}$ & $4(13.3)$ & & & & $12(40.0)$ \\
\hline II & $14(46.7)$ & & & & $12(40.0)$ \\
\hline III & $12(40.0)$ & & & & $6(20.0)$ \\
\hline \multicolumn{6}{|l|}{ Treatment side effects, $n(\%)$} \\
\hline Asthenia & & & $1(3.3)$ & & \\
\hline Chest pain & & $1(3.3)$ & & & \\
\hline Constipation & & $2(6.7)$ & $2(6.7)$ & $2(6.7)$ & \\
\hline Dyspnea & & $1(3.3)$ & & & \\
\hline Epigastric distress & & $3(10.0)$ & $1(3.3)$ & $1(3.3)$ & $1(3.3)$ \\
\hline Headache & & $1(3.3)$ & & & \\
\hline Pruritus & & $1(3.3)$ & & & \\
\hline Tachycardia & & $1(3.3)$ & & & \\
\hline
\end{tabular}

I-MPSS 10-items Italian Mainz Pain-Staging System, IQR interquartile range, MCS mental component summary, NRS Numerical Rating Scale, PSC psychometrically based physical component summary, $S D$ standard deviation, $S F-12$ 12-items health survey

a Median (IQR)

Initial doses of PR tapentadol were established on the basis of pain therapy prior to the study, while dose escalations were dictated by the reported pain intensity at follow-ups. Mean daily dose PR tapentadol at
V90 was roughly $50 \%$ higher than that at V0, indicating a satisfactory titrating process over time. In some cases the daily dose prescribed at V0 was relatively high $(300 \mathrm{mg})$ and did not follow the recommended titration method 
(starting doses of $50 \mathrm{mg}$ 12-hourly); this was due to the switch done in patients already taking high doses of strong opioids, but with intolerable side effects.

High analgesic efficacy was shown in all patients with rapid and significant pain intensity reduction which was observed even after 10 days. Pain intensity reduction further increased and remained stable over time. These results are in agreement with other studies in which PR tapentadol was used to treat CP in patients with arthritis and lumbago [14, 25, 28]. Most patients with such pathologies require lasting pain therapy. Indeed, the majority of our patients reported moderate/severe pain lasting over 12 months. In our study, PR tapentadol showed significant analgesic efficacy over a period of 3 months, and thus may be considered efficacious to treat lasting $\mathrm{CP}$ in such patients. Reported side effects were limited, moderate, and transitory and did not induce patients to quit treatment.

Quality of life, functional, physical, mental, and social issues are key concerns in the treatment of CP. Improvement of the latter is an essential goal of $\mathrm{CP}$ pharmacological management [4, 5]. Given the long-lasting features of $\mathrm{CP}$, long-term pain treatments need to take these issues into account and produce analgesia, limited adverse effects, satisfactory QoL, and physical and mental performance. In our sample, using the SF-12 questionnaire, PCS, and MCS summaries showed improvement over time. Interestingly, improvements were significant for PCS, but not for MCS; however, MCS showed a reduction of IQR over time, thus qualitatively and indirectly demonstrating an improvement trend. PCS and MCS differences between V0 and V90 were significant for men, but not for women, and among patients of $>70$ years (PCS). Widespread CP (as in roughly $50 \%$ of our sample) has been reported to be associated with poor healthrelated QoL and an increased risk of reporting poor SF-12 MCS/PCS scores [29]. This was explained by the presence of psychosocial risk factors such as anxiety and depression for MCS scores, and illness behavior, somatic symptoms, depression, and sleep problems for PCS scores. While illness behavior predicts both poor MCS and PCS scores in CP patients, high levels of psychological distress and anxiety are associated specifically with poor MCS scores. For low-back $\mathrm{CP}$, illness behavior has been reported to affect both mental and physical health-related QoL; when pain is perceived as a threatening symptom it tends to be catastrophized, resulting in increased disability and depression [30]. In our sample, even though the underlying $\mathrm{CP}$ condition and possible physical and psychosocial risk factors persisted over 3 months, an improvement trend for PCS (and more limited for MCS) scores was shown.

Finally, the proportion of patients with relatively high chronicity stage (II and III 87 $\%)$ was reduced over time; at V90 most patients were classified at relatively low chronicity stage (I and II $80 \%$ ). The I-MPSS is a ten-item interview-administered, multi-dimensional measure of pain chronicity. It grades $\mathrm{CP}$ in terms of four pain-related axes: persistence, spreading, medication, and healthcare utilization; it classifies patients at three chronicity stages (I mild; II moderate; and III severe). These stages represent different phases in the chronification process: the higher the stage, the more extensive management interventions will be needed and the less likely a full recovery from CP will be achieved. Chronicity staging has been also suggested as a tool to refer patients with CP to appropriate specialists [23, 31]. Our results show that the PR tapentadol 12-week treatment had a positive effect on the 
patients' chronicity stage, and thus it might have indirectly reduced both pain persistence, medications, and healthcare use.

\section{Limitations}

The study's sample size was relatively small and no comparator was used. Because of the limited number of available patients and similar trials in the literature, no power analysis could have been reported and no standards were prospectively considered. Indeed, this study was designed as a first step towards a future trial in which the long-term efficacy and tolerability profile of PR tapentadol in patients with non-cancer CP will be compared with other opioids. Thus, the reported study was intended to convey an exploratory analysis in order to gather clinical information, to validate the set-up of the trial, and to determine an estimate of the variability of the measurements. Based on this study, a new randomized and controlled study can now be more carefully planned with a satisfactory power analysis and an adequate sample size. The sample size also limited the stratification of the enrolled cases in the gender and age subgroups. To avoid analysis type II error, due to limited cell size, along with tentative quantitative analyses, descriptive and qualitative analyses for these variables were also reported, and hence they should be considered as potential findings. Given the importance of age and gender in $\mathrm{CP}$, these findings justify further research. Multiple comparisons and the number of univariate analyses made in this study may increase the risk of Type I error. To allow interpretation, the level of $P$ value for each analysis was reported. As many of these values were of high significance $(P<0.01)$ this risk is low. Given the consecutive nature of the screened cases and the lack of dropouts, the sample realistically represents our daily practice. The external validity of this report comes from its strong relevance in the practice of pain medicine and the ability to highlight important clinical outcomes in daily clinical practice.

\section{CONCLUSION}

The results of this study add further evidence to the satisfactory efficacy and tolerability profile of lasting PR tapentadol in outpatients with non-cancer CP. Along with efficacious analgesia and limited and tolerable side effects it showed an improvement in QoL and in functional, physical, mental, and social issues in patients with $\mathrm{CP}$. The results are robust enough to warrant a subsequent study with a larger sample and longer observation time.

\section{ACKNOWLEDGMENTS}

No funding or sponsorship was received for this study or publication of this article. All authors discussed the results and commented on the manuscript. All named authors meet the International Committee of Medical Journal Editors (ICMJE) criteria for authorship for this manuscript, take responsibility for the integrity of the work as a whole, and have given final approval for the version to be published.

Conflict of interest. Boaz G. Samolsky Dekel, Sivia Ghedini, Alberto Gori, Alessio Vasarri, GianFranco Di Nino, and Rita M. Melotti declare no conflict of interest.

Compliance with ethics guidelines. The study was approved and authorized by the hospital's ethics committee. All procedures followed were in accordance with the ethical standards of the responsible committee on 
human experimentation (institutional and national) and with the Helsinki Declaration of 1975, as revised in 2000 and 2008, and was conducted according to the International Association for the Study of Pain (IASP)'s guidelines for pain research in animals and humans. All participants were informed of the study aims and structure and that participation was voluntary, anonymous, and would not affect their care. Informed consent was obtained from all patients for being included in the study.

Open Access. This article is distributed under the terms of the Creative Commons Attribution Noncommercial License which permits any noncommercial use, distribution, and reproduction in any medium, provided the original author(s) and the source are credited.

\section{REFERENCES}

1. Breivik H, Collett B, Ventafridda V, Cohen R, Gallacher D. Survey of chronic pain in Europe: prevalence, impact on daily life, and treatment. Eur J Pain. 2006;10:287-333.

2. Pergolizzi J, Alegre C, Blake D, et al. Current considerations for the treatment of severe chronic pain: the potential for tapentadol. Pain Pract. 2012;12:290-306.

3. Varrassi G, Muller-Schwefe G, Pergolizzi J, et al. Pharmacological treatment of chronic pain-the need for CHANGE. Curr Med Res Opin. 2010;26:1231-45.

4. Muller-Schwefe G, Jaksch W, Morlion B, et al. Make a CHANGE: optimising communication and pain management decisions. Curr Med Res Opin. 2011;27:481-8.

5. Coluzzi F, Berti M. Change pain: changing the approach to chronic pain. Minerva Med. 2011;102: 289-307.

6. Chaparro LE, Furlan AD, Deshpande A, MailisGagnon A, Atlas S, Turk DC. Opioids compared to placebo or other treatments for chronic low-back pain. Cochrane Database Syst Rev. 2013;8: CD004959.

7. Manchikanti L, Vallejo R, Manchikanti KN, Benyamin RM, Datta S, Christo PJ. Effectiveness of long-term opioid therapy for chronic non-cancer pain. Pain Physician. 2011;14:E133-56.

8. Raffa RB, Buschmann $\mathrm{H}$, Christoph $\mathrm{T}$, et al. Mechanistic and functional differentiation of tapentadol and tramadol. Expert Opin Pharmacother. 2012;13:1437-49.

9. Schroder W, Vry JD, Tzschentke TM, Jahnel U, Christoph T. Differential contribution of opioid and noradrenergic mechanisms of tapentadol in rat models of nociceptive and neuropathic pain. Eur J Pain. 2010;14:814-21.

10. Schroder W, Tzschentke TM, Terlinden R, et al. Synergistic interaction between the two mechanisms of action of tapentadol in analgesia. J Pharmacol Exp Ther. 2011;337:312-20.

11. Tzschentke TM, Christoph T, Kogel B, et al. (-)-(1R,2R)-3-(3-dimethylamino-1-ethyl-2-methylpropyl)-Phenol hydrochloride (tapentadol $\mathrm{HCl}$ ): a novel mu-opioid receptor agonist/norepinephrine reuptake inhibitor with broad-spectrum analgesic properties. J Pharmacol Exp Ther. 2007;323:265-76.

12. Tzschentke TM, Jahnel U, Kogel B, et al. Tapentadol hydrochloride: a next-generation, centrally acting analgesic with two mechanisms of action in a single molecule. Drugs Today (Barc). 2009;45:483-96.

13. Afilalo M, Etropolski MS, Kuperwasser B, et al. Efficacy and safety of tapentadol extended release compared with oxycodone controlled release for the management of moderate to severe chronic pain related to osteoarthritis of the knee: a randomized, double-blind, placebo- and active-controlled phase III study. Clin Drug Investig. 2010;30:489-505.

14. Afilalo M, Morlion B. Efficacy of tapentadol ER for managing moderate to severe chronic pain. Pain Physician. 2013;16:27-40.

15. Baron R, Kern U, Muller M, Dubois C, Falke D, Steigerwald I. Effectiveness and tolerability of a moderate dose of tapentadol prolonged release for managing severe, chronic low back pain with a neuropathic component: an open-label continuation arm of a randomized phase $3 b$ study. Pain Pract. 2014. (Epub ahead of print).

16. Baron R, Martin-Mola E, Muller M, Dubois C, Falke D, Steigerwald I. Effectiveness and safety of tapentadol prolonged release (PR) versus a combination of tapentadol PR and pregabalin for the management of severe, chronic low back pain 
with a neuropathic component: a randomized, double-blind, phase 3b study. Pain Pract. 2014. (Epub ahead of print).

17. Buynak R, Shapiro DY, Okamoto A, et al. Efficacy and safety of tapentadol extended release for the management of chronic low back pain: results of a prospective, randomized, double-blind, placeboand active-controlled Phase III study. Expert Opin Pharmacother. 2010;11:1787-804.

18. Kress HG, Koch ED, Kosturski H, et al. Tapentadol prolonged release for managing moderate to severe, chronic malignant tumor-related pain. Pain Physician. 2014;17:329-43.

19. Lange B, Kuperwasser B, Okamoto A, et al. Efficacy and safety of tapentadol prolonged release for chronic osteoarthritis pain and low back pain. Adv Ther. 2010;27:381-99.

20. Mercadante S, Porzio G, Ferrera P, et al. Tapentadol in cancer pain management: a prospective openlabel study. Curr Med Res Opin. 2012;28:1775-9.

21. Schwartz S, Etropolski M, Shapiro DY, et al. Safety and efficacy of tapentadol ER in patients with painful diabetic peripheral neuropathy: results of a randomized-withdrawal, placebo-controlled trial. Curr Med Res Opin. 2011;27:151-62.

22. Wild JE, Grond S, Kuperwasser B, et al. Long-term safety and tolerability of tapentadol extended release for the management of chronic low back pain or osteoarthritis pain. Pain Pract. 2010;10:416-27.

23. Samolsky Dekel BG, Gori A, Vasarri A, Adversi M, Di NG, Melotti RM. Psychometric properties and validation of the Italian version of the Mainz pain staging system as a tool for pain-patients referral selection. J Eval Clin Pract. 2014;20:622-30.

24. Hartrick C, Van Hove I, Stegmann JU, Oh C, Upmalis D. Efficacy and tolerability of tapentadol immediate release and oxycodone $\mathrm{HCl}$ immediate release in patients awaiting primary joint replacement surgery for end-stage joint disease: a 10-day, phase III, randomized, double-blind, activeand placebo-controlled study. Clin Ther. 2009;31:260-71.

25. Steigerwald I, Muller M, Davies A, et al. Effectiveness and safety of tapentadol prolonged release for severe, chronic low back pain with or without a neuropathic pain component: results of an open-label, phase 3b study. Curr Med Res Opin. 2012;28:911-36.

26. Steigerwald I, Muller M, Kujawa J, Balblanc JC, CalvoAlen J. Effectiveness and safety of tapentadol prolonged release with tapentadol immediate release on-demand for the management of severe, chronic osteoarthritis-related knee pain: results of an open-label, phase 3b study. J Pain Res. 2012;5:121-38.

27. Steigerwald I, Schenk M, Lahne U, Gebuhr P, Falke D, Hoggart B. Effectiveness and tolerability of tapentadol prolonged release compared with prior opioid therapy for the management of severe, chronic osteoarthritis pain. Clin Drug Investig. 2013;33:607-19.

28. Steigerwald I, Muller M, Kujawa J, Balblanc JC, Calvo-Alen J. Effectiveness and safety of tapentadol prolonged release with tapentadol immediate release on-demand for the management of severe, chronic osteoarthritis-related knee pain: results of an open-label, phase $3 \mathrm{~b}$ study. J Pain Res. 2012;5:121-38.

29. Nicholl BI, Macfarlane GJ, Davies KA, Morriss R, Dickens C, McBeth J. Premorbid psychosocial factors are associated with poor health-related quality of life in subjects with new onset of chronic widespread pain-results from the EPIFUND study. Pain. 2009;141:119-26.

30. Vlaeyen JW, Linton SJ. Fear-avoidance and its consequences in chronic musculoskeletal pain: a state of the art. Pain. 2000;85:317-32.

31. Nicholas MK. When to refer to a pain clinic. Best Pract Res Clin Rheumatol. 2004;18:613-29. 